H. YANAGIHARA

KODAI MATH. J.

11 (1988), $44-46$

\title{
EXTREMAL FUNCTIONS FOR BLOCH CONSTANTS
}

\author{
BY HiROSHI YANAGIHARA
}

\section{Introduction.}

Let $f(z)$ be an analytic function in the unit disc $\Delta=\{|z|<1\}$ with $f^{\prime}(0) \neq 0$. We denote by $r_{f}(z)$ the radius of the largest schlicht disc with center at $f(z)$ in the Riemann image surface of $f: \Delta \rightarrow \mathbf{C}$ (the complex plane). We also denote by $\tilde{r}_{f}(z)$ the radius of the largest disc which is contained in $f(\Delta) \subset \mathbf{C}$. Define $r_{f}$ and $\tilde{r}_{f}$ by

$$
\begin{aligned}
& r_{f}=\sup _{z \in \Delta} r_{f}(z), \\
& \tilde{r}_{f}=\sup _{z \in \Delta} \tilde{r}_{f}(z),
\end{aligned}
$$

respectively. Let $\mathcal{A}$ be a family of all analytic functions $f$ in $\Delta$ with $f^{\prime}(0) \neq 0$ and $A_{0}$ be a family of all analytic functions in $\Delta$ with $f^{\prime}(z) \neq 0, z \in \Delta$. Let $\mathcal{S}$ be a family of all univalent analytic functions in $\Delta$. Then the Bloch, Landau, locally univalent Bloch and univalent Bloch constants are defined respectively by

$$
\begin{aligned}
B & =\inf _{f \in \mathcal{A}} \frac{r_{f}}{\left|f^{\prime}(0)\right|}, \\
\mathcal{L} & =\inf _{f \in \mathcal{A}} \frac{\tilde{r}_{f}}{\left|f^{\prime}(0)\right|}, \\
B_{0} & =\inf _{f \in \mathcal{A}_{0}} \frac{r_{f}}{\left|f^{\prime}(0)\right|}, \\
B_{1} & =\inf _{f \in \mathcal{S}} \frac{r_{f}}{\left|f^{\prime}(0)\right|} .
\end{aligned}
$$

For terminologies our basic references are [3] and [4].

The purpose of the present article is to show the following;

THEOREM 1. Let $f(z)$ be one of the extremal function for the Bloch, Landau, locally univalent Bloch and univalent Bloch constants. Then

$$
\left|\lim _{\varepsilon \rightarrow 0} \frac{1}{\pi} \int_{\varepsilon<|z|<1} \frac{\overline{f^{\prime}(z)}}{z^{2} f^{\prime}(z)} d x d y\right| \leqq 1
$$

Received September 2, 1987 
holds.

\section{Proof of the Theorem.}

Let $f(z)$ be one of the extremal function for the Bloch, Landau, locally univalent Bloch and univalent Bloch constants. Let $t$ be a sfficiently small complex parameter and define an affine transformation $\phi_{t}(z)$ by

$$
\phi_{t}(z)=z+b t \bar{z} .
$$

We shall deform the image $f(\Delta)$ by $\phi_{t}$ as follows. Define $\mu_{t} \in L^{\infty}(\Delta)$ by

$$
\mu_{t}(z)=\frac{\partial\left(\phi_{t} \circ f\right)}{\partial \bar{z}}(z) / \frac{\partial\left(\phi_{t} \circ f\right)}{\partial z}(z) .
$$

Then there exists a unique quasi-conformal automorphism $g_{t}$ of $\bar{\Delta}$ with fixed points 0 and 1 satisfying $\partial g_{t} / \partial \bar{z}=\mu_{t} \partial g_{t} / \partial z$ almost everywhere. Set $f_{t}=\phi_{t} \circ f \circ g_{t}^{-1}$. An easy calculation shows that the Beltrami coefficient of $f_{t}$ vanishes almost everywhere. Hence $f_{t}$ is analytic in $\Delta$. It is clear that $f_{t}(\Delta)=\phi_{t}(f(\Delta))$ and that $f_{t}(z) \rightarrow f(z)$ locally uniformly in $\Delta$, as $t \rightarrow 0$.

LEMMA 1. The asymptotic expansion of the $f_{t}^{\prime}(0)^{-1}$ is given by;

$$
\begin{aligned}
\frac{1}{\left|f_{t}^{\prime}(0)\right|^{2}}= & \frac{1}{\left|f^{\prime}(0)\right|^{2}}\left[1-2 \Re\left\{\frac{b t}{\pi} \lim _{\varepsilon \rightarrow 0} \int_{\varepsilon<|z|<1} \frac{\overline{f^{\prime}(z)}}{z^{2} f^{\prime}(z)} d x d y\right\}\right] \\
& +O\left(t^{2}\right),
\end{aligned}
$$

as $t \rightarrow 0$.

For the proof see [7, Section 3 and 6]. Define $r_{t}$ by

$$
r_{t}=\left\{\begin{array}{l}
\tilde{r}_{f_{t}}, \text { if } f \text { is extremal for the Landau constant, } \\
r_{f_{t}}, \text { otherwise. }
\end{array}\right.
$$

Since the maximum modulus of the eigen values of $\phi_{t}$ is

$$
\left|\frac{\partial \phi_{t}}{\partial z}(z)\right|+\left|\frac{\partial \phi_{t}}{\partial \bar{z}}(z)\right|=1+|b t|
$$

it is clear that

$$
r_{t} \leqq(1+|b t|) r_{0}
$$

By the extremality of $f$, the inequality

$$
\begin{aligned}
\left\{\frac{r_{0}}{\left|f^{\prime}(0)\right|}\right\}^{2} & \leqq\left\{\frac{r_{t}}{\left|f_{t}^{\prime}(0)\right|}\right\}^{2} \\
& \leqq\left\{\frac{r_{0}}{\left|f^{\prime}(0)\right|}\right\}^{2}\left[1+2 \Re\left\{|b t|-\frac{b t}{\pi} \lim _{\varepsilon \rightarrow 0} \int_{\varepsilon<|z|<1} \frac{\frac{f^{\prime}(z)}{z^{2} f^{\prime}(z)}}{d x d y\}}\right]\right.
\end{aligned}
$$




$$
+O\left(t^{2}\right)
$$

holds for sufficiently small $|t|$. Thus we have

$$
\Re\left\{|b t|-\frac{b t}{\pi} \lim _{\varepsilon \rightarrow 0} \int_{\varepsilon<|z|<1} \frac{\overline{f^{\prime}(z)}}{z^{2} f^{\prime}(z)} d x d y\right\}+O\left(t^{2}\right) \geqq 0,
$$

as $t \rightarrow 0$. Since we can choose a complex constant $b$ arbitrarily, we have the desired result.

\section{REFERENCES}

[1] L.V. AhLFors, Lectures on Quasi Conformal Mappings, Princeton, N. J., Van Nostland, 1966.

[2] L.V. Ahlfors and H. Grunsky, Über die Blochsche Konstante, Math. Z., 42 (1937), 671-673.

[3] C.D. MindA, Bloch Constants, J. D'Analyse Math., 41 (1982), 54-84.

[4] Ch. Pommerenke, On Bloch functions, J. London Math. Soc. (2) 2 (1970), 689-695.

[5] A. YAMADA, Q.C. variations for analytic functions (unpublished note).

[6] A. YAMADA, Bloch constant and variation of branch points, Kodai Math. Journal, 9 (1986), 401-405.

[7] H. YanagihaRA, Quasi-conformal variations and local minimality of the AhlforsGrunsky function, J. D'Analyse Math., to appear.

Department of Mathematics

FACUlTy OF SCIENCE

YAMAGUCHI UNIVERSITY

Yoshida, YamaGUCHI 753

JAPAN 